\title{
Tertiary wastewater treatment using bioreactors-clarifiers
}

\author{
Evgeniy Voitov ${ }^{1}$,Yuri Skolubovich ${ }^{1 *}$, Victor Kravchenko ${ }^{2}$ and Tamara Khalturina ${ }^{2}$ \\ ${ }^{1}$ Novosibirsk State University of Architecture and Civil Engineering (Sibstrin), Leningradskaya, 113, \\ Novosibirsk, 630008, Russia \\ ${ }^{2}$ Siberian Federal University, Svobodny Ave., 23 (A), Krasnoyarsk, 660041, Russia
}

\begin{abstract}
The use of technical oxygen instead of air in biological treatment plants can increase their oxidizing ability. Bioreactor-clarifier is a new technical device for tertiary wastewater treatment with the use of oxygen. The design, operation principle and research results of the bioreactor-clarifier for tertiary treatment of urban wastewater are given The use of bioreactors-clarifiers for tertiary treatment of urban wastewater using dissolved technical oxygen allows intensifying the process of biochemical oxidation of organic substances in a contact weighed load and increasing the efficiency of wastewater treatment by BOD and other components.
\end{abstract}

\section{Introduction}

Currently, the issue of using treated domestic wastewater in industrial water supply systems is an urgent one due to the shortage of tap water. However, the quality of wastewater treatment does not allow supplying treated wastewater to the circulating and industrial water supply systems without prior preparation. One way to solve this problem is to introduce an additional stage of wastewater treatment after traditional mechanical and biological treatment, so-called tertiary treatment.

The most widespread in world and domestic practice are methods of filtering treated wastewater using quick filters and contact clarifiers, which are similar in design to the filters used for water treatment.

However, more than half a century of experience in operating these facilities revealed several of their serious shortcomings. These include the rapid colmatization of the filters and contact clarifiers' loading material, which reduces their productivity, as well as the high (up to $15 \%$ ) consumption of purified water for the load regeneration, in addition, the low cleaning effect on the biochemical and chemical water oxygen demand (BOD and COD), the inability to remove nutrients from wastewater.

Previous studies have shown that the processes of dissolved organics biochemical oxidation in the granular layer of the filter loading are characterized by the constants $\mathrm{K} 1=$ $0.05-0.11$ day- 1 and are similar to processes in conventional biooxidants at low BOD values [1]. With a change in the saturation density of the sediment charge, the total oxygen consumption increases, mainly spent on sludge respiration and the processes of biochemical

\footnotetext{
"Corresponding author: michmacha@mail.ru
} 
oxidation of dissolved organics in water are inhibited. Anaerobic zones may appear in the filter loading during the filter cycle and the quality of the treated water will deteriorate, since the oxygen content in the source water is limited.

The purpose of these studies is to develop and test a bioreactor of a new design, which has several advantages compared to existing filters of tertiary treatment of urban wastewater.

The acceleration of the biochemical oxidation process of organic substances is achieved by aeration of water with air, for example in aeration tanks and biofilters [2-5]. Wastewater is aerated with air in a receiving tank to a concentration of $5-7 \mathrm{mg} / \mathrm{dm}^{3}$, before being fed to tertiary treatment filters (bioreactors) at the wastewater treatment plant in Voznesensk, Moscow Region. Bioreactor filters make it possible to achieve a cleaning quality that meets the standards of allowable discharge for fishery reservoirs for suspended solids, $\mathrm{BOD}_{\text {comp }}$, ammonium nitrogen, nitrite nitrogen, COD, oil products and a number of heavy metals [6]. In Russia, it was proposed to intensify the process of organic substances oxidation in filters of tertiary wastewater treatment at "Oksipor" design.

Air is introduced into the upward flow of the filtered fluid through the distribution pipes in order to saturate the water with oxygen in such filters [7]. The obtained results of our experimental studies show, the passage of air bubbles through the filter charge causes resonance cavitation in the pore volumes of the load, which prevents the formation and fixing of the precipitate's structure in the filter body and reduces the cleaning efficiency [8].

In stationary filter loads of quick filters and contact clarifiers, so-called stagnant zones create under conditions of grains soaking by liquid around those near the grains' contact points that reduce free surface and make hydraulic filtering resistance. According to the theoretical calculations and experimental studies, the volume of stagnant zones can reach $37 \%$ of the layer porosity even for spherical particles, which reduces the filters performance [9].

The use of technical oxygen instead of air in biological treatment plants allows increasing their oxidizing ability and the specific oxidation rate by BOD by 5-6 times [3]. However, this technology has not been implemented in filtration facilities of tertiary wastewater treatment.

\section{Materials and methods}

The efficiency increasing of the tertiary treatment facilities for urban wastewater was achieved in a constructively new coagulation water treatment facility - a bioreactorclarifier, which had been developed at the Novosibirsk State University of Architecture and Civil Engineering (Sibstrin) [10].

The scheme of Bioreactor-clarifier work (Figure 1).

Traditional technologies for natural water preparation into drinking water are not designed to purify the original water with color over 120 degrees.

The fact is the supersaturation degree of water by the coagulants hydrolysis products at their usual doses in the metastable zone is insufficient for the solid phase nuclei in the free volume of septic tanks at low temperatures. 


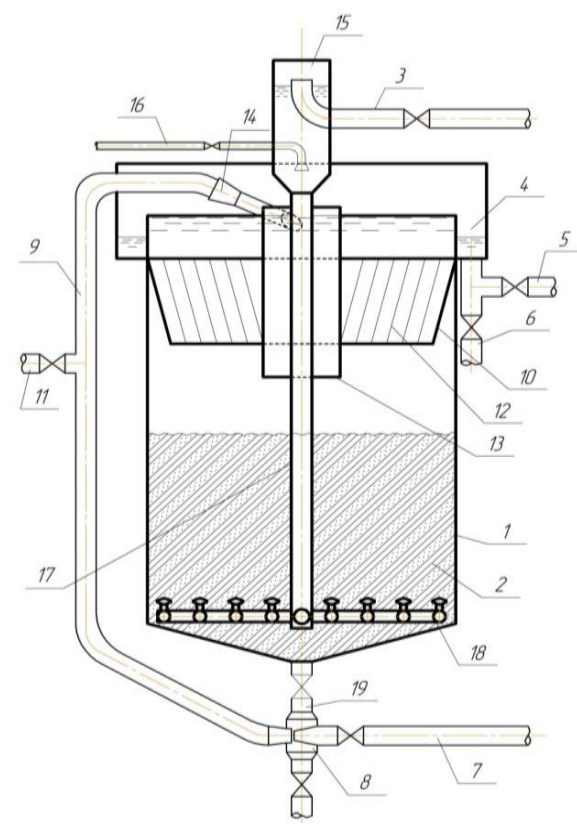

Fig. 1. The scheme of Bioreactor-clarifier work

Initial water is supplied to the air separator 15 through the water supply pipe for clarification 3, where it is saturated with oxygen introduced through the pipe 16. The air separator 15 prevents gas bubbles from entering the suspended contact charge layer. Oxygen-saturated water enters the lower part of the housing 1 through the vertical downpipe 17 , it is evenly distributed over the area of the device's clarification zone with a distribution cap system 18, and it passes upward through the contact loading layer 2 at a speed that allows it to be weighed.

Quartz sand or any other fine-grained material that meets the requirements for mechanical strength and chemical resistance is used as contact load 2. In the suspended contact load layer due to biosorption, sediment is retained and accumulated. Favorable conditions are created for the occurrence of biochemical oxidation processes with a sufficient amount of oxygen dissolved in water.

A part of the sediment flakes is removed from the layer, but it is retained by the thinlayer module 12. It slides down the inclined walls of its rings and settles on the surface of the contact load.

As a result, a high effect of water purification according to the biological and chemical oxygen demand (BOD and COD) is achieved, which is caused both by the content of suspended and dissolved organic substances, so the productivity of the device is increased. The clarified, biochemically purified water is collected by the gutter 4 and discharged by a pipe 5 from the reactor. The contact load is washed in the following sequence, after the protective action time of the weighted contact load expires and the quality of the clarified water deteriorates. The valve on the drainage pipe for washing water 6 opens, and on the drainage pipe for clarified water 5 it closes.

Gate valves are opened on the pipe 19 connecting the pulp supply ejector 8 to the lower part of the device body and on the supply pipe 7 to the wash water ejector 8 . The supply of the clarified water through the pipeline 3 , through the air separator 15 , the vertical lowering pipe 17 and the distribution cap system 18 into the contact mass 2 , continues. 
Flushing water is supplied to the ejector 8 . The contaminated contact weighed load in the form of a pulp is sucked into the ejector of the pulp 8 through a pipe 19 from the bottom of the device and it is transported to the upper part of the device through a pipe 9.

Then, it is tangentially introduced into the semi-submersible cylinder 13 below through an air ejector 14 water level at an angle to its surface.

The contact charge taking out in the ejector 8 is regulated by means of a valve installed on the pipe 19. Air introduced into the pulp is released into the water in the form of bubbles using the ejector 14 and air-water washing of the contact charge occurs, enhanced by the separation of sediment particles and the contact charge grains in the centrifugal field forces in the semi-submersible cylinder 13.

The washed grains of the contact load are deposited in the suspended layer, and the sediment is discharged with the used wash water. The contact load removal in the collection gutter 4 is prevented by a thin-layer module 12 .

The valves on the pipelines 19, 7, 6 are closed after the washing process. The supply of washing water to the ejector 8 is stopped and the device continues to purify the water again. Contact weighed loading is used repeatedly during the cleaning process. If it is necessary to replace the contact weighed load or empty the water treatment device, the pulp is discharged from it through the pipe 11.

The pilot plant included a bioreactor-clarifier, structurally corresponding to the figure 1. The device was filled with fine sand of contact loading of $0.5 \div 0.7 \mathrm{~mm}$ fraction from crushed burned rock. Biologically treated municipal wastewater from the gutter after secondary settling tanks was introduced into the device's air separator.

Oxygen was also supplied to the air trap with a concentration of 7-10 mg / L of treated water. In the air separator, water was freed from the released air and oxygen bubbles, flowed down the vertical lowering pipe and distributed through the cap system into the layer of contact weighed load. The optimal load expansion was $12 \%$. Water was clarified in a suspended layer of contact loading and in a thin-layer module, moving from the bottom to the top.

At the same time, the main part of the sediment was released from the water, organic sludge particles were sorbed on the sediment, and water was clarified to the established norm of $3 \mathrm{mg} / \mathrm{L}$ in terms of suspension content and $6 \mathrm{mg} / \mathrm{L}$ in accordance with $\mathrm{BOD}_{5}$ in the loading layer.

Filter cycles ended within the quality of the treated water became below normal. Part of the sediment, which was not retained in the contact loading layer, was allocated in a thinlayer module in the form of large dense flakes, flowed down and settled on the surface of the contact mass layer.

Up to $30 \%$ of the total decrease in $\mathrm{BOD}_{5}$ in the load did not occur due to the release of suspended solids, but as a result of physicochemical and biochemical processes in the contact mass with a sufficient amount of dissolved oxygen in water.

Siberian surface water and groundwater are characterized by low temperatures for a long year period. Many groundwater sources' organic composition are formed of soil and peat humus, marsh feeding of rivers; decomposition of plankton, higher water and soil grass in reservoirs and lakes.

Organic colloids in natural waters and in humic substances give a water color. It's yellowish coloration of varying intensity.

\section{$3 \quad$ Results}

The bioreactor-clarifier can be simplified as a kind of filter, where upward filtration is realized through a layer of suspended (pseudo fluidized) loading in a state of the so-called dense phase with a relative volume concentration of its particles of more than 0.3 . 
In this case, there is visually no movement of the loading grains with their circulation along the layer height, observed during water washing of filters and contact clarifiers. Due to the stagnant zones absence in the load and the increase in its porosity in the bioreactorclarifier, the dirt capacity and the working filtration rate increase up to 1.5 times, which ensures a corresponding increase in the structure productivity.

An increase in the structure productivity, referred to the unit of its production space, reduces building size for the equipment, construction cost and operating costs. The use of ejection washing of the reactor reduces the consumption of washing water by up to three times, and increases the overall useful productivity of the wastewater treatment plant.

The use of bioreactors-clarifiers for tertiary treatment of urban wastewater using dissolved technical oxygen allows intensifying the process of biochemical oxidation of organic substances in a contact weighed load and increasing the efficiency of wastewater treatment by BOD and other components

\section{Discussing}

The use of the proposed bioreactor-clarifier for tertiary wastewater after water biological treatment allowed an almost 1.5 -fold increase in the rate of upward flow in its charge compared to contact clarifiers, as well as an increase in the cleaning efficiency not only in terms of suspended, colloidal, but also dissolved organic substances.

At the end of the daily cycle of water treatment, the contact mass was washed in the upper part of the device with its transportation by the pulp feed ejector and tangentially introduced through the air ejector into the semisubmersible cylinder to clean and isolate the contact charge particles in the centrifugal forced zone.

The removal of the washable contact charge with the wash water was prevented by a thin-layer module.

The washing provided effective regeneration of the contact load without the accumulation of residual contaminants in it. The pipeline connecting the pulp feed ejector to the lower part of the device body with a valve on it allowed regulating the contact loading of ejector during the washing, disconnecting and sanding the ejector itself and the tangential pulp feed pipe to the upper part of the housing after washing. The use of ejection washing allowed reducing the flow rate of wash water to $5 \%$ out of purified water.

1. A bioreactor-clarifier is developed for the tertiary treatment of urban wastewater, which provides an increase in productivity and work efficiency compared to contact clarifiers and quick filters.

2. The performance of bioreactor clarifiers for tertiary wastewater treatment is 1.5 times higher than the contact clarifiers' performance.

3. Bioreactor-clarifiers increase the cleaning efficiency not only in the content of suspended, colloidal, but also dissolved organic substances.

4. The use of ejection washing of the reactor reduces the consumption of washing water by up to three times, which increases the overall useful productivity of the wastewater treatment plant.

\section{References}

1. E.L. Voytov, Z.P. Nesterenko and A.G. Pchelkin, Energy 9 (2011)

2. C.F. Forster and D.A.J. Wase, Environmental biotechnology. Chichester (2012)

3. Yu.V. Voronov and S.V. Yakovlev, Wastewater and wastewater treatment. Publishing Association of Construction Universities (2017) 
4. V.A Vavilin, V.B Vasiliev and S.V Rytov, Modelling of Organic Matter Destruction by Microorganism Association. Nauka (2013)

5. T. Wik, On modeling the dynamics of fixed biofilm reactors. Chalmers University of Technology (2012)

6. V.B. Smirnov and D. A. Guskov, Plumbing, heating, air conditioning 6 (2017)

7. P. Nechaev and A.S. Slavinsky, Water supply and sanitary engineering 12 (2014)

8. E.L. Voytov and Y.L. Scolubovich, Preparation of drinking water from surface sources with a high natural and anthropogenic pollution. NSUACE (Sibstrin) (2010)

9. L.S. Leibenzon, Movement of natural liquids and gases in a porous medium. State Publishing House of Technical and Theoretical literature (2015)

10. Pat. 2307075 RF, MKI C 02 F 1/52. E.L. Voitov and Y.L. Skolubovich Cleaning Water device. Bul. 27 [5] (2013)

11. T. Wik, On modeling the dynamics of fixed biofilm reactors. Chalmers University of Technology (2011) 